Research Paper

\title{
First-line continual EGFR-TKI plus local ablative therapy demonstrated survival benefit in EGFR-mutant NSCLC patients with oligoprogressive disease
}

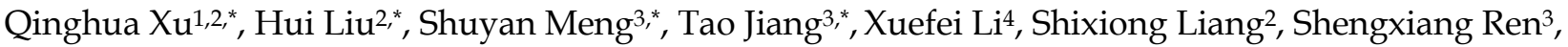 \\ Caicun Zhou ${ }^{3,5,}, \mathbb{}$ \\ 1. Department of Radiation Oncology, Shanghai Pulmonary Hospital, School of Medicine, Tongii University, Shanghai, People's Republic of China \\ 2. Department of Radiation Oncology, Shanghai Pulmonary Hospital, School of Medicine, Soochow University, Suzhou, People's Republic of China \\ 3. Department of Oncology, Shanghai Pulmonary Hospital, School of Medicine, Tongii University, Shanghai, People's Republic of China \\ 4. Department of Lung Cancer and Immunology, Shanghai Pulmonary Hospital, Tongii University School of Medicine, Shanghai, People's Republic of China \\ 5. Department of Oncology, Shanghai Pulmonary Hospital, School of Medicine, Soochow University, Suzhou, People's Republic of China \\ *: Qinghua Xu, Hui liu, Shuyan Meng and Tao Jiang contributed equally to this paper.
}

$\square$ Corresponding author: Prof. Caicun Zhou, Department of Oncology, Shanghai Pulmonary Hospital, Tongji University, 507 Zhengmin Road, Shanghai, 200433, People's Republic of China. E-mail: caicunchow@126.com

(c) Ivyspring International Publisher. This is an open access article distributed under the terms of the Creative Commons Attribution (CC BY-NC) license (https:// creativecommons.org/licenses/by-nc/4.0/). See http://ivyspring.com/terms for full terms and conditions.

Received: 2018.04.04; Accepted: 2018.10.01; Published: 2019.01.01

\begin{abstract}
Introduction: The effect of local ablative therapy (LAT) for oligoprogressive epidermal growth factor receptor (EGFR) mutation non-small cell lung cancer (NSCLC) remains undetermined. This study aimed to investigate the survival benefit of addition of LAT to EGFR-TKIs in EGFR-mutant NSCLC patients with oligoprogression during TKI therapy.

Materials and Methods: Patients with stage IIIB/IV EGFR mutant NSCLC who had oligoprogressive disease during the first-line EGFR-TKI therapy from March 2011 to February 2016 were identified. The primary research point were progression-free survivall (PFSI), defined as time of initiation of TKI therapy to Response Evaluation Criteria in Solid Tumours (RECIST) 1.1 defined progress disease (PD) or death and PFS2, defined as time of initiation of TKI therapy to off-TKI PD. The second research piont inclued overal survival (OS) and safety.

Results: A total of 206 patients were included. The median follow-up time was 42 months (20.0-69.6 months). The median PFS1, median PFS2 and median OS for the related cohort were 10.7 months ( $95 \% \mathrm{Cl}, 10.1-13.3$ months), 18.3 months ( $95 \% \mathrm{Cl}, 17.4-19.2$ months) and 37.4 months ( $95 \%$ $\mathrm{Cl}, 35.9-38.9$ months) respectively. Survival rates of 1 year, 2 years and 3 years were $94.1 \%, 78.9 \%$, and $54.7 \%$, respectively. Multivariate analysis revealed that female, EGFR exon 19 mutation, one metastatic lesion, partial or complete response to prior EGFR TKIs therapy were the independent prognostic factors. No unexpected toxicities were observed.

Conclusion: The current study suggested that the addition of LAT to EGFR-TKI could provide satisfactory survival benefit for EGFR-mutant NSCLC patients with oligoprogression during first-line EGFR-TKI treatment.
\end{abstract}

\section{Introduction}

Lung cancer is the leading cause of cancer-related death worldwide.1-2 Non-small cell lung cancer (NSCLC) accounts for more than $80 \%$ of all cases. Epidermal growth factor receptor (EGFR) plays a critical role in cancer cells proliferation and survival. EGFR activating mutations occur more frequently in Asian patients compared with Caucasian patients. ${ }^{3-5}$ Landmark clinical trials have 
demonstrated the superior progression-free survival (PFS) and quality-of-life of EGFR tyrosine kinase inhibitors (TKIs) over the standard platinum-based doublet chemotherapy in NSCLC patients with EGFR activating mutations.6-12 Several EGFR TKIs (gefitinib, erlotinib, icotinib and afatinib) have been the standard first-line therapy in patients with EGFR-mutant NSCLC. However, acquired resistance would occur in patients who initially responded to EGFR TKI, after median PFS of $10-14$ months. ${ }^{6-12}$ Radiological progression does not always imply that all metastatic sites share the same cause of resistance. Some tumor sites might still be sensitive to EGFR TKI beyond Response Evaluation Criteria in Solid Tumors (RECIST) disease progression. The potential tumor heterogeneity suggests that continuation of EGFR TKIs may provide additional benefit in patients with slow progression. ${ }^{13-15}$

The oligoprogressive disease represents an indolent status of patients with advanced disease who are receiving active systemic therapy but with limited number of metastatic sites showing disease progression. Local treatment to all oligoprogressive lesions is thought to eradicate the de-differentiated clones and restore sensitivity of the metastatic disease. ${ }^{16-18}$ For patients with oligoprogressive disease harboring EGFR mutation and treated with EGFR TKI, whether the addition of local ablative therapy (LAT) could extend the survival benefit in oligoprogressive disease remains undetermined. To address this issue, we performed this study to investigate the outcomes of EGFR-mutant NSCLC patients treated with continual EGFR TKI and LAT after oligoprogression.

\section{Materials and Methods}

\section{Patients cohort}

We conducted a retrospective study on patients with stage IIIB/IV EGFR-mutant NSCLC who had oligoprogressive disease during the first-line EGFR-TKI therapy from March 2011 to February 2016 at Shanghai Pulmonary Hospital. Recruited patients met the following criteria: they had diagnosis of pathologically confirmed NSCLC with confirmed activating EGFR mutation (exon 19 deletion or exon 21 L858R mutation), with stage IIIB/IV disease according to the 7 th edition of the American Joint Committee on Cancer staging system, were 18 years or older, with an Eastern Cooperative Oncology Group (ECOG) performance status (PS) score of 2 or less, had received EGFR-TKI as first-line therapy, with five or fewer metastases, not including the primary tumor during the first-line EGFR-TKI therapy. The oligoprogressive lesions were radically treated with
LATs. Oligometastatic disease was defined as the presence of $\leq 5$ lesions in 1 to multiples organs during EGFR TKI therapy. Never-smoker was defined as a person who had smoked fewer than 100 cigarettes during his/her lifetime. Patients received continuous EGFR-TKI therapy until symptomatic extracranial progression, rapid progression, worsening ECOG PS or life-threatening complications as examples of those who may not be suitable for continued TKI therapy or clinicians believed that patients could not benefit from continuation of TKI therapy. The treatment response was evaluated 6-8 weeks after the initiation of therapy and then every 2 months according to RECIST 1.1. The EGFR-TKIs used in this study included gefitinib (250 $\mathrm{mg}$, once a day), erlotinib (150 $\mathrm{mg}$, once a day), and icotinib (125 mg, three times a day). EGFR mutations were tested by an amplification refractory mutation system as described in our previous studies (Amoy Diagnostics Co., Ltd., Xiamen, China). ${ }^{19}$ All mutational analyses were performed at the Thoracic Cancer Institute, Tongji University Medical School, Shanghai, People's Republic of China.

Baseline characteristics were recorded from electronic records by retrospective collection, including age at diagnosis (taken at date of diagnostic biopsy), sex, smoking status, ECOG PS, tumor histology prior therapy, treatment, oligometastatic disease and number of oligometastatic sites. Routine surveillance imaging includes chest computed tomography (CT), abdomen CT and brain magnetic resonance imaging. Patients underwent bone scan when suspected for bone metastasis, and positron emission tomography scan when suspected for systemic progression.

\section{Statistical analysis}

Descriptive statistics were used to summarise patient characteristics by treatment group. PFS1 was calculated from time of initiation of TKI therapy to first RECIST 1.1 defined progress disease (PD). PFS2 was calculated from time of initiation of TKI therapy to off-TKI PD. Overal survival (OS) was calculated from the date of lung cancer diagnosis to death from any cause or was censored at the last follow-up date. Kaplan-Meier curve and 2-sided log-rank test were used for univariate survival analyses. The Cox proportional hazards model was used for uni- and multivariate survival analyses to calculate the hazard ratio (HR) and corresponding 95\% confidence intervals. Subgroup analysis was also carried out with age, gender, ECOG PS, hitology, Disease stage, metastases number, mutation type, EGFR TKIs, reponse to EGFR TKIs in 6 months and oligoprogressive symptom as the stratification factors. $P$ values were 2 -sided and considered significant if $<$ 
0.05. All statistical analyses were performed using the SPSS statistical software, version 22.0 (IBM Corp., Armonk, NY).

\section{Results}

\section{Patient Characteristics}

A total of 206 patients with oligoprogressive diseases harboring EGFR mutations were included, who were treated with first-line EGFR-TKI within the study period. The patient characteristics were presented in Table 1 . The majority of patients $(174 / 206,84.5 \%)$ had histology of adenocarcinoma and the median age was 58 years. Briefly, $58.3 \%$ of patients were female, $74.3 \%$ had ECOG PS 0 or 1 , $81.1 \%$ had stage IV disease, $60.7 \%$ were never smokers, $38.3 \%$ had only one site metastases, $56.8 \%$ had exon 21 L858R mutation and $68.0 \%$ had partial or complete response.

Table 1. Baseline patient characteristics.

\begin{tabular}{|c|c|c|}
\hline Characteristic & Patients (n) & $(\%)$ \\
\hline Median age, y (range) & $58(28 \sim 83)$ & \\
\hline$<65$ & 143 & 69.4 \\
\hline$\geq 65$ & 63 & 30.6 \\
\hline \multicolumn{3}{|l|}{ Gender } \\
\hline Male & 97 & 41.7 \\
\hline Female & 109 & 58.3 \\
\hline \multicolumn{3}{|l|}{ ECOG performance status } \\
\hline $0 \sim 1$ & 153 & 74.3 \\
\hline 2 & 53 & 25.7 \\
\hline \multicolumn{3}{|l|}{ Histology } \\
\hline Adenocarcinoma & 174 & 84.5 \\
\hline Squamous cell & 2 & 0.9 \\
\hline Large cell & 7 & 3.4 \\
\hline Adeno-squamous & 14 & 6.8 \\
\hline NOS & 9 & 4.4 \\
\hline \multicolumn{3}{|l|}{ Disease stage } \\
\hline IIIB & 39 & 18.9 \\
\hline IV & 167 & 81.1 \\
\hline \multicolumn{3}{|l|}{ Smoking status } \\
\hline Non-smoker & 125 & 60.7 \\
\hline Present or former smoker & 81 & 39.3 \\
\hline \multicolumn{3}{|l|}{ Metastases number } \\
\hline 1 & 79 & 38.3 \\
\hline 2 & 25 & 12.1 \\
\hline 3 & 31 & 15.1 \\
\hline 4 & 42 & 20.4 \\
\hline 5 & 29 & 14.1 \\
\hline \multicolumn{3}{|l|}{ EGFR mutation } \\
\hline Exon 19 deletion & 89 & 43.2 \\
\hline Exon $21 \mathrm{~L} 858 \mathrm{R}$ & 117 & 56.8 \\
\hline \multicolumn{3}{|l|}{ EGFR TKIs } \\
\hline Gefitinib & 107 & 51.9 \\
\hline Erlotinib & 47 & 22.8 \\
\hline Icotinib & 52 & 25.3 \\
\hline \multicolumn{3}{|c|}{ Response to first-line EGFR TKIs } \\
\hline Partial or complete response & 140 & 68.0 \\
\hline Stable disease & 41 & 19.9 \\
\hline Disease progression & 25 & 12.1 \\
\hline \multicolumn{3}{|l|}{ Oligoprogressive symptom } \\
\hline Symptomatic & 71 & 34.5 \\
\hline Asymptomatic & 135 & 65.5 \\
\hline \multicolumn{3}{|c|}{ Second- or further-line treatment } \\
\hline Chemotherapy & 164 & 79.6 \\
\hline Osimertinib \pm chemotherapy & 11 & 5.4 \\
\hline
\end{tabular}

\begin{tabular}{|c|c|c|}
\hline СТHM & 31 & 15.0 \\
\hline \multicolumn{3}{|l|}{ Metastasis location } \\
\hline Brain & 124 & 60.2 \\
\hline Bone & 86 & 41.7 \\
\hline Adrenal & 35 & 17.0 \\
\hline Lung & 40 & 19.4 \\
\hline Liver & 18 & 8.7 \\
\hline Chest wall & 10 & 4.9 \\
\hline Neck lymph nodes & 9 & 4.4 \\
\hline Intestine & 1 & 0.5 \\
\hline \multicolumn{3}{|l|}{ LAT for oligometastasis } \\
\hline Brain & 124 & \\
\hline Whole-brain irradiation & 23 & 18.5 \\
\hline SRS & 89 & 71.8 \\
\hline Surgery + whole-brain irradiation & 12 & 9.7 \\
\hline Bone & 86 & \\
\hline Surgery + EBRT (30 Gy) & 4 & 4.7 \\
\hline EBRT (30-40 Gy) & 82 & 95.3 \\
\hline Adrenal & 35 & \\
\hline Surgery & 10 & 28.6 \\
\hline SBRT & 16 & 45.7 \\
\hline EBRT (45-50 Gy) & 9 & 25.7 \\
\hline Lung & 40 & \\
\hline SBRT & 26 & 65.0 \\
\hline EBRT (55-63 Gy) & 11 & 27.5 \\
\hline Surgery & 3 & 7.5 \\
\hline Liver & 18 & \\
\hline Radiofrequency ablation & 16 & 88.9 \\
\hline Surgery & 2 & 11.1 \\
\hline Chest wall & 10 & \\
\hline EBRT (45-55Gy) & 10 & 100.0 \\
\hline Neck lymph nodes & 9 & \\
\hline EBRT (55-63Gy) & 9 & 100.0 \\
\hline Intestine & 1 & \\
\hline Surgery & 1 & 100.0 \\
\hline \multicolumn{3}{|c|}{$\begin{array}{l}\text { Abbreviations: ECOG, Eastern Cooperative Oncology Group; NOS, not otherwise } \\
\text { specified; EGFR, epidermal growth factor receptor; TKI, tyrosine kinase inhibitor. }\end{array}$} \\
\hline
\end{tabular}

$124(60.2 \%)$ cases of Oligoprogressive diseases were identified in brain, $86(41.7 \%)$ in bone, 35 (17.0\%) in adrenal glands, $18(8.7 \%)$ in liver, $40(19.4 \%)$ in lung, $10(4.9 \%)$ in chest wall, $9(4.4 \%)$ in neck lymph nodes, and $1(0.5 \%)$ in intestine. Only $32(15.5 \%)$ patients underwent surgical treatment, including 12 cases of brain, 10 cases of adrenal glands, 4 cases of bone, 3 cases of lung, 2 cases of liver, 1 case of intestine. $107(51.9 \%)$ patients received first-line gefitinib therapy, 47 (22.8\%) received erlotinib therapy and $52(25.3 \%)$ received icotinib. 118 (57.3\%) patients received second-line platinum-based combination chemotherapy, 46 (22.3\%) patients received single-agent chemotherapy and 31 (15.1\%) patients received Chinese traditional herbal medicine (CTHM) treatment. Only $11(5.3 \%)$ patients received second or third-line treatment of third-generation EGFR-TKI osimertinib (Table 1).

\section{PFS and OS}

The median follow-up time was 42 months (20.0-69.6 months). The median PFS1 (mPFS1), median PFS2 (mPFS2) and median OS (mOS) for the related cohort were 10.7 months $(95 \% \mathrm{CI}, 10.1-13.3$ months), 18.3 months (95\% CI, 17.4-19.2 months) and 
37.4 months (95\% CI, 35.9-38.9 months), respectively (Figure 1). Survival rates of 1-year, 2-year and 3-year were $94.1 \%, 78.9 \%$, and $54.7 \%$, respectively. For the 124 patients who had brain oligometastases, the mPFS1, mPFS2 and mOS were 11.3 months $(95 \% \mathrm{CI}$, 10.7-11.8 months), 19.0 months (95\% CI, 17.9-20.0 months) and 37.7 months (95\% CI, 36.8-38.6 months), respectively. For the 86 patients who had bone oligometastases, the mPFS1, mPFS2 and mOS were 10.5 months (95\% CI, 9.4-11.6 months), 17.9 months (95\% CI, 16.9-18.9 months) and 36.6 months (95\% CI, 32.5-40.7 months) respectively. For the 35 patients who had adrenal glands oligometastases, the mPFS1, mPFS2 and mOS were 11.4 months (95\% CI, 9.3-13.5 months), 19.5 months (95\% CI, 16.9-22.1 months) and 38.5 months (95\% CI, 34.3-42.7 months) respectively. For the 35 patients who had liver oligometastases, the mPFS1, mPFS2 and mOS were 7.6 months $(95 \% \mathrm{CI}$, 4.1-11.1 months), 13.8 months (95\% CI, 10.9-16.7 months) and 24.7 months (95\% CI, 7.8-41.5 months) respectively. The mPFS1, mPFS2 and mOS for those 32 patients who had surgical treatment were 11.7 months (95\% CI, 9.7-13.7 months), 18.9 months (95\% CI, 16.3-22.5 months) and 39.2 months $(95 \% \mathrm{CI}$, 34.5-43.9 months) respectively. Patients who received gefitinib, erlotinib and icotinib had comparable mPFS1 (10.7, 10.2 and 10.7 months, $P=0.97)$, mPFS2 $(18.8,17.9$ and 18.4 months, $P=0.99)$ and mOS (37.1, 34.7 and 37.8 months, $P=0.96$ ).

\section{Subgroup analysis on PFS1, PFS2 and OS}

Univariate analysis revealed that male patients had significantly shorter mPFS1, mPFS2 and mOS than female patients (mPFS1: HR=0.59, 95\% CI: 0.43-0.77, $P<0.001$, mPFS2: HR=0.55, 95\% CI: 0.41-0.73, $P<0.001$, mOS: HR=0.47, 95\% CI: 0.34-0.64, $P<0.001)$. Patients with adenocarcinoma had significantly longer mPFS1, mPFS2 and mOS than those with other histological types (mPFS1: $\mathrm{HR}=1.94$, 95\% CI: 1.32-2.84, $P=0.001$, mPFS2: $\mathrm{HR}=1.87,95 \% \mathrm{CI}$ : 1.27-2.74, $P=0.001$, mOS: $\mathrm{HR}=1.98,95 \%$ CI: 1.36-2.89, $P<0.001)$. Never-smokers had significantly longer mPFS1, mPFS2 and mOS than former/current-smoker patients (mPFS1: HR=0.69, 95\% CI: 0.52-0.92, $P=$ 0.009, mPFS2: HR=0.68, 95\% CI: 0.50-0.91, $P=0.006$, mOS: HR=0.63, 95\% CI: 0.47-0.86, $P=0.003$ ). Patients with 1 metastatic lesion had significantly better mPFS1, mPFS2 and mOS than patients with more than 1 metastatic lesion (mPFS1: HR $=0.59,95 \% \mathrm{CI}$ : $0.44-0.79, \quad P<0.001$, mPFS2: HR=0.62, 95\% CI: $0.47-0.83, P=0.001$, mOS: HR=0.56, 95\% CI: 0.41-0.76, $P<0.001)$. Patients with EGFR exon 19 deletion had significantly better mPFS1, mPFS2 and mOS than those with EGFR exon 21 L858R (mPFS1: HR=4.93, 95\% CI: 3.53-6.89, $P<0.001$, mPFS2: HR=5.10, 95\% CI:
3.64-7.11, $P<0.001$, mOS: HR=5.4, 95\% CI: 3.77-7.74, $P$ $<0.001)$. Patients who had partial or complete response to first-line EGFR TKIs had significantly better mPFS1, mPFS2 and mOS than patients who had stable disease or disease progression (mPFS1: $\mathrm{HR}=0.34$, 95\% CI: 0.25-0.46, $P<0.001, \mathrm{mPFS} 2$ : $\mathrm{HR}=0.34,95 \%$ CI: $0.25-0.46, P<0.001, \mathrm{mOS}: \mathrm{HR}=0.35$, 95\% CI: 0.25-0.48, $P<0.001$ ) (Figure 2 and Table 2). In multivariate analysis, the variables independently associated with prolonged mPFS1, mPFS2 and mOS were female, EGFR exon 19 mutation, 1 metastatic lesion, partial or complete responses to EGFR TKIs therapy in 6 months (Table 3 ).
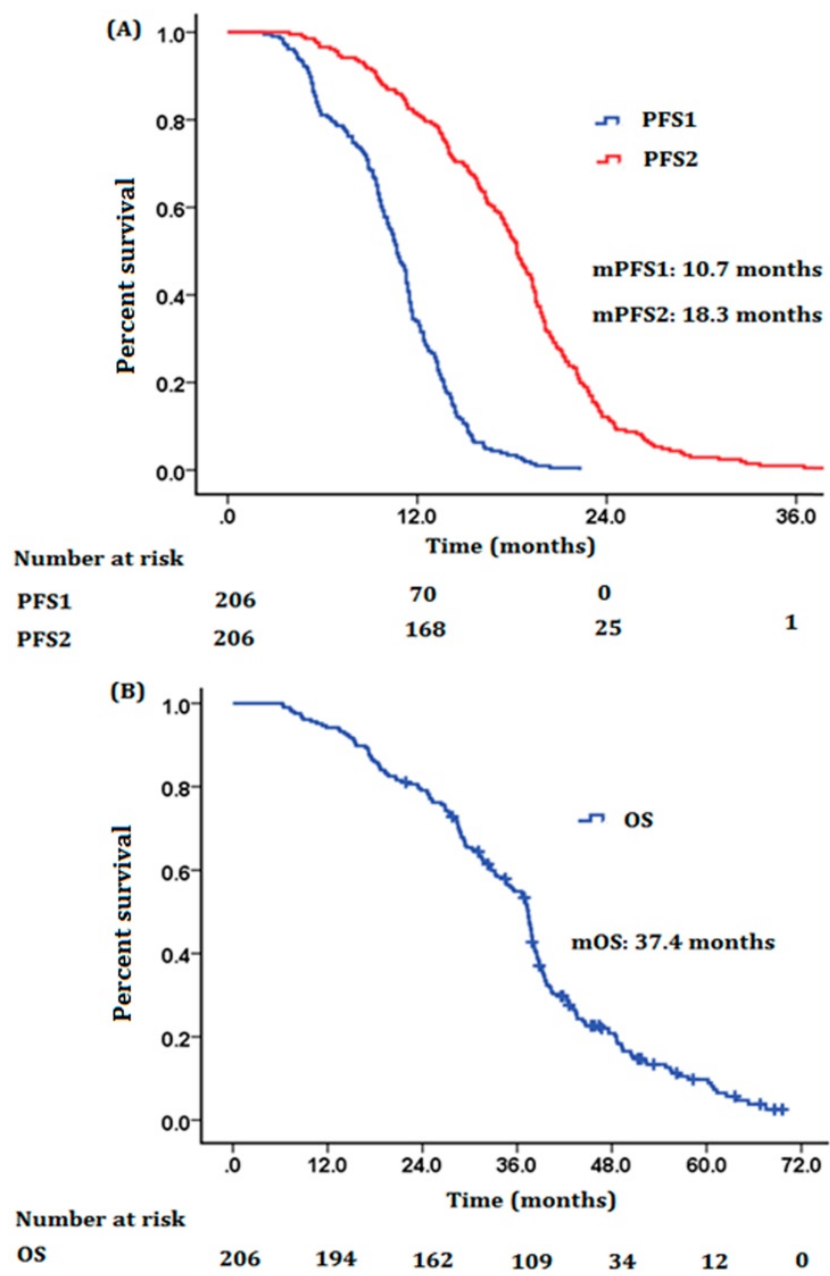

Figure 1. Kaplan-Meier plot of (A) PFS1, PFS2 and (B) OS for all patients in this study cohort. Abbreviations: mPFS, media progression-free survival; mOS, media overal survival.

\section{Toxicity}

The most common toxicities of EGFR TKIs therapy included skin rash, diarrhea, neutropenia, fatigue, nausea, vomiting, and pneumonitis. The majority of toxicities were grade 1 to 2 (G1-2). G3 skin rash occurred in $11(5.3 \%)$ patients. G3 diarrhea occurred in $8(3.9 \%)$ patients. G3 pneumonitis was 
observed in $3(1.5 \%)$ patients. No G4 or G5 toxicity was recorded. The majority of adverse events attributing to LATs occurred to lung. G3 radiation pneumonia was reported in 3 patients within 3 months after SBRT. There was no other documented G3/4 adverse event due to the addition of radiotherapy.

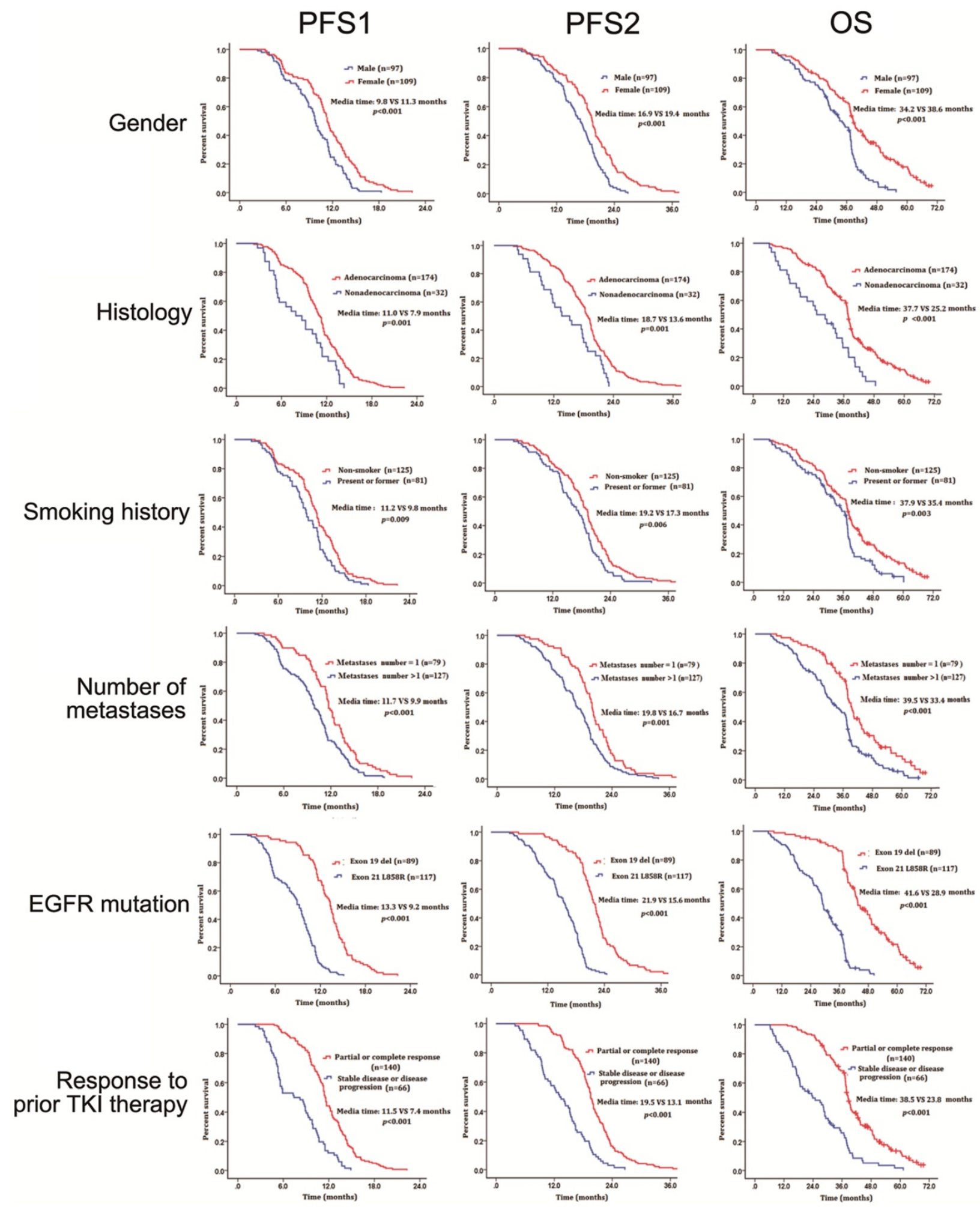

Figure 2. The effect of different clinical factors on survival. Abbreviations: PFS, progression-free survival; OS, overal survival; EGFR, epidermal growth factor receptor; $\mathrm{TKI}$, tyrosine kinase inhibitor. 
Table 2. Univariable analysis of clinical factors potentially associated with PFS and OS.

\begin{tabular}{|c|c|c|c|c|c|c|c|c|c|}
\hline Characteristic & $\begin{array}{l}\text { mPFS1 } \\
\text { (m) }\end{array}$ & HR $(95 \% C I)$ & $p$ Value & $\begin{array}{l}\text { mPFS2 } \\
\text { (m) }\end{array}$ & HR $(95 \% C I)$ & $p$ Value & $\begin{array}{l}\mathrm{mOS} \\
(\mathrm{m})\end{array}$ & HR $(95 \% C I)$ & $p$ Value \\
\hline \multicolumn{10}{|l|}{ Age (year) } \\
\hline$<65$ & 10.8 & \multirow{2}{*}{$\begin{array}{l}1.25 \\
(0.92-1.68)\end{array}$} & \multirow[t]{2}{*}{0.148} & 18.6 & \multirow{2}{*}{$\begin{array}{l}1.26 \\
\mathbf{( 1 . 0 1 - 1 . 6 3 )}\end{array}$} & \multirow[t]{2}{*}{0.092} & 37.6 & \multirow{2}{*}{$\begin{array}{l}1.29 \\
\mathbf{( 0 . 9 6 - 1 . 7 4 )}\end{array}$} & \multirow[t]{2}{*}{0.131} \\
\hline$\geq 65$ & 10.5 & & & 17.4 & & & 37.1 & & \\
\hline \multicolumn{10}{|l|}{ Gender } \\
\hline Male & 9.8 & \multirow{2}{*}{$\begin{array}{l}0.59 \\
\mathbf{( 0 . 4 3 - 0 . 7 7 )}\end{array}$} & \multirow{2}{*}{$<0.001$} & 16.9 & \multirow{2}{*}{$\begin{array}{l}0.55 \\
\mathbf{( 0 . 4 1 - 0 . 7 3 )}\end{array}$} & \multirow[t]{2}{*}{$<0.001$} & 34.2 & \multirow{2}{*}{$\begin{array}{l}0.47 \\
\mathbf{( 0 . 3 4 - 0 . 6 4 )}\end{array}$} & \multirow{2}{*}{$<0.001$} \\
\hline Female & 11.3 & & & 19.4 & & & 38.6 & & \\
\hline \multicolumn{10}{|l|}{ ECOG performance status } \\
\hline $0 \sim 1$ & 10.7 & \multirow{2}{*}{$\begin{array}{l}1.08 \\
(0.79-1.49)\end{array}$} & \multirow[t]{2}{*}{0.614} & 18.4 & \multirow{2}{*}{$\begin{array}{l}1.06 \\
\mathbf{( 0 . 7 7 - 1 . 4 5 )}\end{array}$} & \multirow[t]{2}{*}{0.739} & 37.5 & \multirow{2}{*}{$\begin{array}{l}0.99 \\
\mathbf{( 0 . 7 1 - 1 . 3 8 )}\end{array}$} & \multirow[t]{2}{*}{0.745} \\
\hline 2 & 10.7 & & & 18.3 & & & 37.3 & & \\
\hline \multicolumn{10}{|l|}{ Histology } \\
\hline Adenocarcinoma & 11.0 & \multirow{2}{*}{$\begin{array}{l}1.94 \\
\mathbf{( 1 . 3 2 - 2 . 8 4 )}\end{array}$} & \multirow{2}{*}{0.001} & 18.7 & \multirow{2}{*}{$\begin{array}{l}1.87 \\
\mathbf{( 1 . 2 7 - 2 . 7 4 )}\end{array}$} & \multirow{2}{*}{0.001} & 37.7 & \multirow{2}{*}{$\begin{array}{l}1.98 \\
\mathbf{( 1 . 3 6 - 2 . 8 9 )}\end{array}$} & \multirow[t]{2}{*}{$<0.001$} \\
\hline Nonadenocarcinoma & 7.9 & & & 13.6 & & & 25.2 & & \\
\hline \multicolumn{10}{|l|}{ Disease stage } \\
\hline IIIB & 11.5 & 1.38 & 0.068 & 19.4 & 1.35 & 0.096 & 38.4 & 1.43 & 0.069 \\
\hline IV & 10.5 & $(0.97-1.96)$ & & 18.2 & $(0.95-1.91)$ & & 37.2 & $(0.97-2.10)$ & \\
\hline Smoking status & & & & & & & & & \\
\hline Non-smoker & 11.2 & 0.69 & 0.009 & 19.2 & 0.68 & 0.006 & 37.9 & 0.63 & 0.003 \\
\hline Present or former smoker & 9.8 & $(0.52-0.92)$ & & 17.3 & $(0.51-0.90)$ & & 35.4 & $(0.47-0.86)$ & \\
\hline Metastases number & & & & & & & & & \\
\hline 1 & 11.7 & 0.59 & $<0.001$ & 19.8 & 0.62 & 0.001 & 39.5 & 0.56 & $<0.001$ \\
\hline$>1$ & 9.9 & $(0.44-0.79)$ & & 16.7 & $(0.47-0.83)$ & & 33.4 & $(0.41-76)$ & \\
\hline EGFR mutation & & & & & & & & & \\
\hline Exon 19 deletion & 13.3 & 4.93 & $<0.001$ & 21.9 & 5.1 & $<0.001$ & 41.6 & & $<0.001$ \\
\hline Exon $21 \mathrm{~L} 858 \mathrm{R}$ & 9.2 & $(3.53-6.89)$ & & 15.6 & $(3.64-7.11)$ & & 28.9 & $(3.77-7.74)$ & \\
\hline Response to first-line EGFR TKIs & & & & & & & & & \\
\hline Partial or complete response & 11.5 & 0.34 & $<0.001$ & 19.5 & 0.34 & $<0.001$ & 38.5 & 0.35 & $<0.001$ \\
\hline Stable disease or disease progression & 7.4 & $(0.25-0.46)$ & & 13.1 & $(0.25-0.46)$ & & 23.8 & $(0.25-0.48)$ & \\
\hline Oligoprogressive symptom & & & & & & & & & \\
\hline Symptomatic & 10.7 & 0.98 & 0.550 & 16.7 & 0.85 & 0.080 & 34.2 & 0.91 & 0.215 \\
\hline Asymptomatic & 10.8 & $(0.70-1.36)$ & & 18.4 & $(0.58-1.12)$ & & 37.6 & $(0.62-1.30)$ & \\
\hline
\end{tabular}

Table 3. Multivariable analysis of covariables associated with PFS and OS.

\begin{tabular}{|c|c|c|c|c|c|c|}
\hline \multirow[t]{2}{*}{ Variable } & \multicolumn{2}{|l|}{ PFS1 } & \multicolumn{2}{|l|}{ PFS2 } & \multicolumn{2}{|l|}{ OS } \\
\hline & HR (95\% CI) & $p$ Value & HR (95\% CI) & $p$ Value & HR $(95 \%$ CI $)$ & $p$ Value \\
\hline Gender (male VS. female) & $\begin{array}{l}0.69 \\
\mathbf{( 0 . 5 0 - 0 . 9 5 )}\end{array}$ & 0.025 & $\begin{array}{l}0.61 \\
(0.43-0.82)\end{array}$ & 0.016 & $\begin{array}{l}0.612 \\
(0.43-0.87)\end{array}$ & 0.006 \\
\hline $\begin{array}{l}\text { Histology (adenocarcinoma vs. } \\
\text { nonadenocarcinoma) }\end{array}$ & $\begin{array}{l}1.27 \\
(0.83-1.94)\end{array}$ & 0.281 & $\begin{array}{l}0.85 \\
(0.55-1.30)\end{array}$ & 0.445 & $\begin{array}{l}1.70 \\
\mathbf{( 1 . 1 0 - 2 . 6 2 )}\end{array}$ & 0.016 \\
\hline $\begin{array}{l}\text { Smoking status (non-smoker vs. present or } \\
\text { former) }\end{array}$ & $\begin{array}{l}1.08 \\
(0.78-1.51)\end{array}$ & 0.648 & $\begin{array}{l}0.95 \\
\mathbf{( 0 . 6 9 - 1 . 3 1 )}\end{array}$ & 0.753 & $\begin{array}{l}1.02 \\
(0.72-1.44)\end{array}$ & 0.916 \\
\hline EGFR mutation (exon19 vs. exon 21 ) & $\begin{array}{l}4.06 \\
(2.86-5.76)\end{array}$ & $<0.001$ & $\begin{array}{l}3.90 \\
(2.75-5.98)\end{array}$ & $<0.001$ & $\begin{array}{l}4.68 \\
(3.19-6.87)\end{array}$ & $<0.001$ \\
\hline Metastases number (1 vs. $>1$ ) & $\begin{array}{l}1.64 \\
\mathbf{( 1 . 1 9 - 2 . 1 6 )}\end{array}$ & 0.002 & $\begin{array}{l}0.642 \\
(0.48-0.86)\end{array}$ & 0.003 & $\begin{array}{l}1.76 \\
\mathbf{( 1 . 2 8 - 2 . 4 0 )}\end{array}$ & $<0.001$ \\
\hline $\begin{array}{l}\text { Response to first-line EGFR TKIs (Partial or } \\
\text { complete response vs. Stable disease or disease } \\
\text { progression) }\end{array}$ & $\begin{array}{l}2.92 \\
(1.37-2.69)\end{array}$ & $<0.001$ & $\begin{array}{l}0.49 \\
(\mathbf{0 . 3 6 - 0 . 6 9 )}\end{array}$ & $<0.001$ & $\begin{array}{l}1.93 \\
(\mathbf{1 . 3 8}-\mathbf{2 . 7 0 )}\end{array}$ & $<0.001$ \\
\hline
\end{tabular}

Abbreviations: HR, hazard ratio; CI, confidence interval; PFS, progression-free survival; OS, overal survival; EGFR, epidermal growth factor receptor; TKI, tyrosine kinase inhibitor.

\section{Discussion}

Almost all patients receiving EGFR-TKI therapy will eventually experience disease progression. Premature discontinuation of EGFR TKI therapy may result in rapid progression of symptoms and tumor regrowth, while reintroduction of TKI therapy could slow tumor growth again. ${ }^{20}$ Preclinical data indicated that coexistence of EGFR-dependent and EGFR-independent clones arising in vitro from the same environmental stress. ${ }^{21}$ On removal of selective pressure by discontinuation of EGFR-TKI therapy, the TKI-sensitive clones may regrow and result in rapid
PD with the potential risk of flare-up of symptoms. Therefore, continue EGFR-TKI treatment beyond RECIST progression may prolong survival benefits in clinically selected patients. ASPIRATION has demonstrated a potential improvement in median PFS with continuation of erlotinib beyond progression. ${ }^{22}$ However, the characteristics of those patients who continued erlotinib therapy and those who did not were not balanced. Significantly more patients who had recurrent disease, ECOG PS 0 or 1 at PFS1, longer median PFS1, improved depth of response, and a longer median time from best overall response to PFS1 continued erlotinib therapy than those who did 
not. IMPRESS reported that continuation of gefitinib after radiological disease progression on first-line gefitinib did not prolong PFS in patients who received platinum-based doublet chemotherapy as subsequent line of treatment. ${ }^{23}$ Therefore, the patient population who could benefit from continuing EGFR-TKI therapy remained controversial.

Patients who developed local or slow/minimal progression (oligoprogression) during EGFR-TKI treatment present unique clinical characteristics which could benifit from continual EGFR-TKI plus LAT. Lo ${ }^{13}$ et al showed that continuation of EGFR-TKI therapy plus locoregional treatment (surgery and radiotherapy) after PD, delayed the need for second-line chemotherapy by 3 months. In a retrospective analysis by Weickhardt 24 et al, 25 paitents (15 ALK positive cases, 10 EGFR mutant cases) continuing TKI therapy with LAT beyond PD significantly extended PFS. However, the sample size of these studies was too small. To our knowledge, this is one of the largest single institutional analyses in the literature of NSCLC patients with EGFR mutant who developed oligoprogresson and received LAT during first-line EGFR-TKI therapy. The median PFS1 from the study was consistent with previous studies of first-line EGFR-TKI therapy in EGFR mutation-positive NSCLC. ${ }^{6-12}$ The median PFS2 was 18.3 months and the median OS was 37.4 months in this whole cohort, which were similar to those observed in the retrospective studies. ${ }^{24-26}$ The results of mPFS2 and mOS in the current study were longer than that in the ASPIRATION study (14.1 months in median PFS2, 31.0 months in median OS), ${ }^{22}$ suggesting that first-line EGFR TKIs therapy plus LATs might provide better outcome in EGFR-mutant NSCLC patients who had oligoprogressive disease. Therefore, continual EGFR TKI as systemic treatment plus additional LAT for progressive sites may be appropriate in EGFR-mutant patients with oligoprogresson in clinical practice.

With the development of TKI that target T790M mutation, the optimal strategy and timing for switching treatment will be an important challenge ${ }^{26}$ Although LAT seemed to be effective for EGFR-mutant patients with oligoprogression, the criteria of patient selection remained uncertain. To identify the subgroup of patients who can benefit the most along with less toxicities become a controversial topic. In our study, only 11 (5.3\%) patients who had T790M mutation received second or third-line osimertinib treatment after re-biopsy, and had achieved a mOS of 41.2 months. The following osimertinib treatment in patient with T790M mutation could be delayed by extending the duration of treatment with EGFR-TKI plus LAT, which contributed to a longer mPFS and mOS. Whether or not asymptomatic oligoprogression requires ongoing local treatment remains controversial. In many retrospective studies, continuing EGFR-TKI therapy in combination with local therapy for isolated CNS disease or non-CNS tumors in selected patients could be beneficial. ${ }^{25}$, 28-29 In our study, patients with symptomatic oligoprogression had marginally worse PFS2 than those with asymptomatic oligoprogression. Our study suggested that patients should immediately recieved LAT with asymptomatic or symptomatic oligoprogression.

There are several limitations that should be acknowledged. Firstly, this is a single arm retrospective study in a single institutional, which inevitably results in a selection bias. Secondly, this study did not use predefined treatment strategies according to the different progression pattern types. The actual treatment strategy utilized with regard to continuing EGFR TKI treatment plus LAT or switching to a cytotoxic chemotherapy or third-generation EGFR TKI after the RECIST-PD assessment was at the discretion of the physician. Thirdly, re-biopsy was not performed in most patients to investigate possible mechanisms of acquired resistance such as the presence of EGFR T790M mutation, MET amplification, or transformation to small cell lung cancer. Perhaps most importantly, we do not have a comparator to judge the true benefit from EGFR-TKIs continuation treatment or LAT.

This study provided rationale for considering the approach of continuation of first-line EGFR-TKI therapy plus LAT in EGFR-mutant NSCLC patients after oligoprogression. Combination of EGFR TKI plus LAT could provide additional benefits for these patients. Further multicenter, prospective studies are needed to confirm the benefit of continuation of first-line EGFR TKI and LAT after oligoprogression.

\section{Acknowledgements}

This study was supported by Shanghai Science and Technology Commission Guiding Project (16411964400) and Shanghai Municipal Health and Family Planning Commission Foundation (201440385).

\section{Competing Interests}

The authors have declared that no competing interest exists.

\section{References}

1. Siegel RL, Miller KD, Jemal A. Cancer Statistics, 2017. CA Cancer J Clin. 2017;67:7-30

2. Chen W, Zheng R, Baade PD, et al. Cancer statistics in China, 2015. CA Cancer J Clin. 2016:66:115-1132.

3. Rosell R, Moran T, Queralt C, et al. Screening for epidermal growth factor receptor mutations in lung cancer. N Engl J Med. 2009;361:958-967. 
4. Shigematsu H, Lin L, Takahashi T, et al. Clinical and biological features associated with epidermal growth factor receptor gene mutations in lung cancers. J Natl Cancer Inst. 2005;97(5):339-346.

5. Wu YL, Zhong WZ, Li LY, et al. Epidermal growth factor receptor mutations and their correlation with gefitinib therapy in patients with non-small cell lung cancer: a meta-analysis based on updated individual patient data from six medical centers in mainland China. J Thorac Oncol. 2007;2:430-439.

6. Mok TS, $\mathrm{Wu} \mathrm{YL}$, Thongprasert S, et al. Gefitinib or carboplatin-paclitaxel in pulmonary adenocarcinoma. N Engl J Med. 2009;361:947-957.

7. Han JY, Park K, Kim SW, et al. First-SIGNAL: first-line single-agent iressa versus gemcitabine and cisplatin trial in never-smokers with adenocarcinoma of the lung. J Clin Oncol. 2012;30:1122-1128.

8. Mitsudomi T, Morita S, Yatabe Y, et al. West Japan Oncology Group. Gefitinib versus cisplatin plus docetaxel in patients with non-small-cell lung cancer harbouring mutations of the epidermal growth factor receptor (WJTOG3405): an open label, randomised phase 3 trial. Lancet Oncol. 2010;11:121-128.

9. Maemondo M, Inoue A, Kobayashi K, et al. North-East Japan Study Group. Gefitinib or chemotherapy for non-small-cell lung cancer with mutated EGFR. N Engl J Med. 2010;362:2380-2388.

10. Zhou C, Wu YL, Chen G, et al. Erlotinib versus chemotherapy as first-line treatment for patients with advanced EGFR mutation-positivenon-small-cell lung cancer (OPTIMAL, CTONG-0802): a multicentre, open-label,randomised, phase 3 study. Lancet Oncol. 2011;12(8):735-742.

11. Shi $\mathrm{Y}$, Zhang $\mathrm{L}$, Liu X, et al. Icotinib versus gefitinib in previously treated advanced non-small-cell lung cancer (ICOGEN): a randomised, double-blind phase 3 non-inferiority trial. Lancet Oncol. 2013;14(10):953-961.

12. Sequist LV, Yang JC, Yamamoto N, et al. Phase III study of afatinib or cisplatin plus pemetrexed in patients with metastatic lung adenocarcinoma with EGFR mutations. J Clin Oncol. 2013;31:3327-3334.

13. Lo PC, Dahlberg SE, Nishino M, et al. Delay of treatment change after objective progression on first-line erlotinib in epidermal growth factor receptor-mutant lung cancer. Cancer. 2015;121:2570-2577.

14. Nishie $K$, Kawaguchi $T$, et al. Epidermal growth factor receptor tyrosine kinase inhibitors beyond progressive disease: a retrospective analysis for Japanese patients with activating EGFR mutations. J Thorac Oncol. 2012;7:1722-1727.

15. Park K, Yu CJ, Kim SW,et al. First-Line Erlotinib Therapy Until and Beyond Response Evaluation Criteria in Solid Tumors Progression in Asian Patients With Epidermal Growth Factor Receptor Mutation-Positive Non-Small-Cell Lung Cancer: The ASPIRATION Study. JAMA Oncol. 2016;2(3):305-312.

16. Campo M, Al-Halabi H, Khandekar M, et al. Integration of Stereotactic Body Radiation Therapy With Tyrosine Kinase Inhibitors in Stage IV Oncogene-Driven Lung Cancer. Oncologist. 2016;21(8):964-973.

17. Basler L, Kroeze SG, Guckenberger M. SBRT for oligoprogressive oncogene addicted NSCLC. Lung Cancer. 2017;106:50-57.

18. Kim C, Hoang CD, Kesarwala AH, et al. Role of Local Ablative Therapy in Patients with Oligometastatic and Oligoprogressive Non-Small Cell Lung Cancer. J Thorac Oncol. 2017;12(2):179-193.

19. Li X, Ren R, Ren S,et al. Peripheral blood for epidermal growth factor receptor mutation detection in non-small cell lung cancer patients. Transl Oncol. 2014;7(3):341-348

20. Riely GJ, Kris MG, Zhao B, et al. Prospective assessment of discontinuation and reinitiation of erlotinib or gefitinib in patients with acquired resistance to erlotinib or gefitinib followed by the addition of everolimus. Clin Cancer Res. 2007;13(17):5150-5155.

21. Kim Y, Ko J, Cui Z, et al. The EGFR T790M mutation in acquired resistance to an irreversible second-generation EGFR inhibitor. Mol Cancer Ther. 2012;11(3):784-791.

22. Park K, Yu CJ, Kim SW, et al. First-Line Erlotinib Therapy Until and Beyond Response Evaluation Criteria in Solid Tumors Progression in Asian Patients With Epidermal Growth Factor Receptor Mutation-Positive Non-Small-Cell Lung Cancer: The ASPIRATION Study. JAMA Oncol. 2016;2(3):305-312

23. Soria JC, Wu YL, Nakagawa K, et al. Gefitinib plus chemotherapy versus placebo plus chemotherapy in EGFR-mutation-positive non-small-cell lung cancer after progression on first-line gefitinib (IMPRESS): a phase 3 randomised trial. Lancet Oncol. 2015;16(8):990-998.

24. Weickhardt AJ, Scheier B, Burke JM,et al. Local ablative therapy of oligoprogressive disease prolongs disease control by tyrosine kinase inhibitors in oncogene-addicted non-small-cell lung cancer. J Thorac Oncol. 2012;7(12):1807-1814

25. Yu HA, Sima CS, Huang J, et al. Local therapy with continued EGFR tyrosine kinase inhibitor therapy as a treatment strategy in EGFR-mutant advanced lung cancers that have developed acquired resistance to EGFR tyrosine kinase inhibitors. J Thorac Oncol. 2013;8(3):346-351.

26. Qiu B, Liang $\mathrm{Y}$, Li O, et al. Local Therapy for Oligoprogressive Disease in Patients With Advanced Stage Non-small-cell Lung Cancer Harboring Epidermal Growth Factor Receptor Mutation. Clin Lung Cancer. 2017;S1525-7304(17)30107-9.

27. Mok TS, Wu Y-L, Ahn M-J, et al. Osimertinib or Platinum-Pemetrexed in EGFR T790M-Positive Lung Cancer. N Engl J Med. 2017;376(7):629-640.

28. Shukuya T, Takahashi T, Naito T, et al. Continuous EGFR-TKI administration following radiotherapy for non-small cell lung cancer patients with isolated CNS failure. Lung Cancer. 2011;74(3):457-461.
29. Conforti F, Catania C, Toffalorio F, et al. EGFR tyrosine kinase inhibitors beyond focal progression obtain a prolonged disease control in patients with advanced adenocarcinoma of the lung. Lung Cancer. 2013;81(3):440-444. 